\title{
Atypical Chemokine Receptors in Cardiovascular Disease
}

\author{
Selin Gencer ${ }^{1, *}$ Emiel P. C. van der Vorst ${ }^{1, *}$ Maria Aslani ${ }^{1} \quad$ Christian Weber ${ }^{1,2,3} \quad$ Yvonne Döring ${ }^{1,2, *}$ \\ Johan Duchene ${ }^{1, *}$
}

${ }^{1}$ Institute for Cardiovascular Prevention (IPEK), LMU Munich, Munich, Germany

${ }^{2}$ German Centre for Cardiovascular Research (DZHK), Partner Site Munich Heart Alliance, Munich, Germany

${ }^{3}$ Cardiovascular Research Institute Maastricht (CARIM), Maastricht University, Maastricht, The Netherlands

Address for correspondence Johan Duchene, PhD, Institute for Cardiovascular Prevention, Ludwig-Maximilians-University Munich, Pettenkoferstr. 8a und 9, D-80336 München, Germany (e-mail: Johan.Duchene@med.uni-muenchen.de).

Yvonne Döring, PhD, Institute for Cardiovascular Prevention, LudwigMaximilians-University Munich, Pettenkoferstr. 8a und 9, D-80336 München, Germany (e-mail: ydoering@med.Imu.de).

Thromb Haemost 2019;119:534-541.
Abstract
Keywords
- atherosclerosis
- cardiovascular diseases
- chemokines
- atypical chemokine receptors

Inflammation has been well recognized as one of the main drivers of atherosclerosis development and therefore cardiovascular diseases (CVDs). It has been shown that several chemokines, small 8 to $12 \mathrm{kDa}$ cytokines with chemotactic properties, play a crucial role in the pathophysiology of atherosclerosis. Chemokines classically mediate their effects by binding to G-protein-coupled receptors called chemokine receptors. In addition, chemokines can also bind to atypical chemokine receptors (ACKRs). ACKRs fail to induce G-proteindependent signalling pathways and thus subsequent cellular response, but instead are able to internalize, scavenge or transport chemokines. In this review, we will give an overview of the current knowledge about the involvement of ACKR1-4 in CVDs and especially in atherosclerosis development. In the recent years, several studies have highlighted the importance of ACKRs in CVDs, although there are still several controversies and unexplored aspects that have to be further elucidated. A better understanding of the precise role of these atypical receptors may pave the way towards novel and improved therapeutic strategies.

\section{Introduction}

Cardiovascular diseases (CVDs) cover a spectrum of conditions that affect the heart and blood vessels, and may manifest in life-threatening events such as myocardial infarction (MI), stroke and aneurysm. The main pathology behind CVDs is atherosclerosis, a lipid-driven, chronic inflammatory disease that impacts arteries. ${ }^{1}$ Atherosclerosis is initiated by haemodynamic shear stress-related damage to the endothelium, increasing the endothelial permeability and in turn its susceptibility to lipid invasion. Subsequent modification of lipids in the intima along with the endothelial damage trigger an inflammatory response in which immune cells infiltrate into the sub-endothelial layer. ${ }^{2}$ This process results

\footnotetext{
* These authors contributed equally to the manuscript and share first or last authorship.
}

in an on-going plaque development within the arterial wall, which may ultimately occlude the blood vessels or rupture leading to atherothrombosis. Arterial occlusion and the resulting hypoxia of downstream tissues (ischaemia) may progress into severe clinical consequences, such as ischaemic stroke, MI or organ dysfunction.

Current state-of-the-art therapy of CVDs is mostly limited to the mitigation of hyperlipidaemia and management of thrombotic factors, for example, use of statins and aspirin, respectively. ${ }^{3}$ In addition to demanding health care procedures causing both economic and social burdens, CVDs still remain the primary cause of mortality worldwide. ${ }^{4}$ This clearly signifies the necessity of novel therapeutic measures which specifically address the main mechanisms regulating atherosclerosis development, such as leukocyte infiltration into atherosclerotic lesions. Chemokines and their receptors

(c) 2019 Georg Thieme Verlag KG Stuttgart · New York
DOI https://doi.org/ $10.1055 / \mathrm{s}-0038-1676988$. ISSN 0340-6245. 
are involved in the onset of atherosclerosis and thereby represent promising potential therapeutic targets.

\section{Chemokines and Their Receptors in Atherosclerosis}

Chemokines, also known as chemotactic cytokines, were first identified to control immune cell migration in the context of inflammation and later in homeostatic conditions. Inflammatory (induced during inflammation) and homeostatic (constitutively expressed in steady state) chemokines bind to Gprotein-coupled receptors (GPCRs). Such chemokine receptors are expressed on all immune cells and their activation by chemokines mediate leukocyte cell migration. However, it is now clear that chemokines can also modulate a plethora of other cellular functions such as proliferation, survival, differentiation, mobilization, cytokine release and phagocytosis in several immune cell populations. ${ }^{5}$ Given their immunologically relevant functions, it is not surprising that chemokines and their receptors extensively contribute to various stages of atherosclerosis, such as leukocyte trafficking and influx into atherosclerotic plaques, as well as proliferation, apoptosis and foam cell formation of different cell types within the lesions. ${ }^{6}$

In addition to these 'classical' chemokine receptors, chemokines also bind to atypical chemokine receptors (ACKRs). Four functional ACKRs have been so far assigned: ACKR1 (also known as [aka] Duffy antigen receptor for chemokine [DARC]), ACKR2 (aka D6), ACKR3 (aka CXCR7) and ACKR4 (aka CCRL1). While ACKR1 and ACKR2 bind inflammatory chemokines, ACKR3 and ACKR4 bind homeostatic chemokines (-Fig. 1). ACKRs are structurally similar to GPCRs but they do not couple to $G$ proteins due to the absence of the Asp-Arg-Tyr-Leu-Ala-Ile-Val (DRYLAIV) motif in the second intracellular loop. ${ }^{7,8}$ As a result, ACKRs fail to induce Gprotein-dependent signalling pathways and subsequent cellular responses, such as chemotaxis. ${ }^{9}$ Instead, ACKRs can internalize, scavenge, transport or present chemokines and thus regulate the bioavailability of chemokines and thereby chemokine signalling via 'classical' receptors. ${ }^{10}$ In contrast to 'classical' receptors, ACKRs are mainly expressed by erythrocytes and non-haematopoietic cells, such as lymphatic and vascular endothelial cells, with the exception of ACKR2 and ACKR3, which are also expressed by some leukocyte subsets.

The rationale behind current research focusing on CVDs is to identify key players in this multifaceted disease to develop suitable therapeutics. Accordingly, ACKRs are of particular interest as they are major regulators of chemokine availability and signalling, thereby potentially contributing to the development of CVDs. In this review, we will discuss the features of each ACKR and their contribution to CVDs, notably in atherosclerosis (-Fig. 1).

\section{ACKR1}

\section{Receptor Characteristics and Signalling}

ACKR1, also known as DARC, binds more than 20 different inflammatory CC and CXC chemokines and was ascribed a unique expression profile in erythrocytes, venular endothe- lial cells and cerebellar Purkinje neurons. On the venular endothelium, ACKR1 can internalize and transport inflammatory chemokines from the tissue onto the luminal endothelial cell surface. ${ }^{11}$ This machinery not only protects soluble chemokines from degradation but also allows their presentation at the surface of endothelial cells and consequently leads to the firm adhesion of circulating leukocytes. Notably, ACKR1 expression is up-regulated in post-capillary venules and veins under inflammation, ${ }^{12}$ suggesting that endothelial ACKR1 plays a crucial role in leukocyte recruitment in inflammatory diseases.

All sub-Saharan Africans and 70\% of African Americans carry the variant rs2814778(G) in the gene encoding ACKR1 making this variant the most predictive ancestry-informative marker of African origin. ${ }^{13}$ The variant corresponds to a single A to $\mathrm{G}$ substitution in the promoter region of ACKR1, which disrupts the binding site for the GATA1 erythroid transcription factor. As a consequence, individuals who are homozygous for the allele specifically lack ACKR1 expression on erythrocytes but still express ACKR1 on endothelium and cerebellum, causing a Duffy-negative phenotype. ${ }^{14}$ We recently found that ACKR1 is highly expressed by nucleated erythroid cells (NECs) in the bone marrow where it regulates the homeostasis of haematopoietic stem and progenitor cells (HSPCs) and controls downstream haematopoiesis. ${ }^{15}$ In the absence of ACKR1, the steadystate haematopoiesis is altered; bone marrow HSPCs localize remotely from NECs and give rise to phenotypically distinct neutrophils. Overall, these findings highlight that ACKR1 expression in the erythroid lineage regulates patterns of haematopoiesis and the ultimate phenotype of myeloid cells. The specific properties of the neutrophils produced in Duffynegative individuals may have a positive impact on innate immune responses against pathogens. On the other hand, a stronger immune response may be detrimental in the context of chronic inflammation and autoimmune disease.

\section{Clinical Significance of ACKR1}

Few studies investigated the role of ACKR1 in atherosclerosis. A study by Wan et al suggested that ACKR1 plays a harmful role in atherosclerosis as the absence of ACKR1 (using conventional global knockout) in $\mathrm{Apoe}^{-1-}$ mice showed a reduction in atherosclerotic plaque size. ${ }^{16}$ Interestingly, although Ackr1 deficiency did not affect plasma cholesterol levels, plaque stability or macrophage content in the atherosclerotic lesions, it was associated with a decreased $\mathrm{T}$ cell number in the aorta. Whether this is due to the capacity of ACKR1 to mediate chemokine transcytosis and leukocyte extravasation remains to be further investigated. In line with this, the expression of ACKR1 was up-regulated in the aorta of $A p o e^{-I-}$ mice fed a high-fat diet (HFD). ${ }^{16}$ As mentioned above, ACKR1 is exclusively expressed on the venular and not arterial (including aorta) vessels. Interestingly, a recent study pointed out that ACKR1 was detected in the vasa vasorum around the aorta of wild-type mice, and its expression in these vessels was increased in the aortas of $A p o e^{-1-}$ mice with atherosclerotic lesions. ${ }^{17}$ It still remains to be defined whether the reduction of T cell infiltration in the aorta of $A c k r 1^{-1-}$ mice was due to a decrease of $\mathrm{T}$ cells in the atherosclerotic plaques or in the 


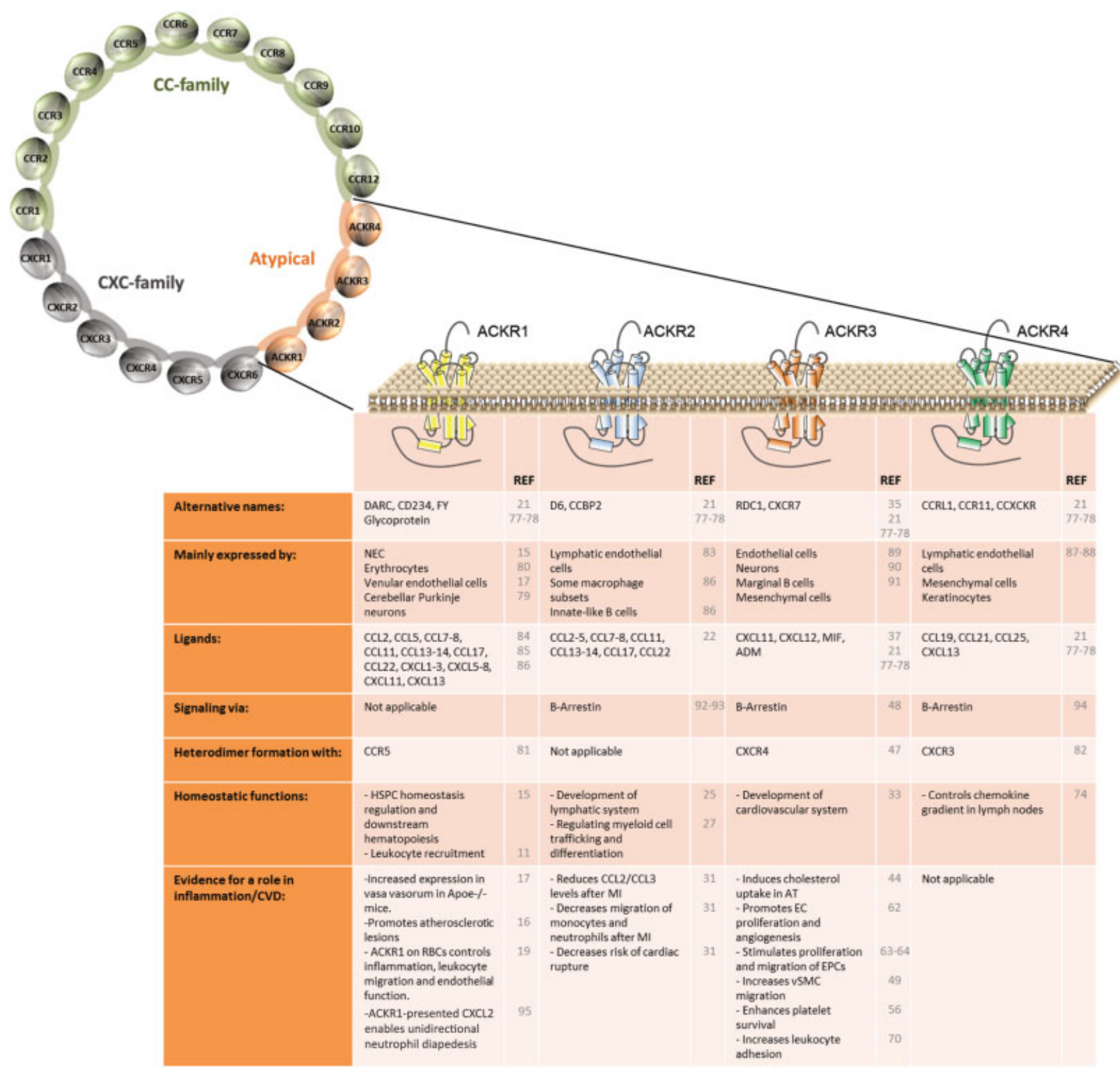

Fig. 1 Overview of expression and functions of the atypical chemokine receptors (ACKRs). The circle displays all currently identified chemokine receptors and depicts the 4 ACKRs (orange) that are further highlighted in the table. Appropriate references for each statement are depicted in the REF columns. ADM, adrenomedullin; AT, adipose tissue; CVD, cardiovascular disease; EC, endothelial cell; EPC, endothelial progenitor cell; HSPC, haematopoietic stem and progenitor cells; MI, myocardial infarction; MIF, macrophage inhibitory factor; NEC, nucleated erythroid cells; $\mathrm{RBC}$, red blood cell; vSMC, vascular smooth muscle cell.

artery tertiary lymphoid organs (ATLOs), which are atherosclerosis-associated lymphoid aggregates participating in $\mathrm{T}$ and $B$ cell responses against atherosclerosis-specific autoantigens. ${ }^{18}$ It would therefore be crucial to determine whether ACKR1 expressed in the vasa vasorum around the aorta is involved in the formation of ATLOs and/or the recruitment of T cells in these lymphoid tissues. Further investigation of $A c k r 1^{-1}$ - mice may shed new light on the role of lymphoid cells in ATLOs which still needs to be clearly defined.

While these studies determined a role of endothelial ACKR1 in atherosclerosis, little is known about erythroid ACKR1 in this context. While it is well-known that HFD induces endothelial dysfunction, a recent study by Unruh et al demonstrated that HFD also affects red blood cell (RBC) function, ${ }^{19}$ notably by increasing the levels of CCL2 bound to RBCs, which promotes interactions between leukocytes and endothelial cells. Importantly, this is partly due to ACKR1 expression on RBCs as pro-inflammatory response levels in RBCs were dramatically reduced in the absence of ACKR1. Therefore, this study not only demonstrates that RBCs play a central role in endothelial dysfunction and leukocyte recruitment in a HFD context, but also highlights the importance of ACKR1 on erythroid cells in atherosclerosis.

Future research on the role of ACKR1 in atherosclerosis should focus on dissecting the specific role of both endothelial and erythroid ACKR1 in the initiation, development and 
consequences of the disease. What is the function of endothelial ACKR1 in ATLOs and subsequently the effects on atherosclerosis? In contrast, how does the absence of ACKR1 on erythroid cells in the bone marrow and the production of phenotypically distinct myeloid cells, affect atherosclerosis development? Individuals of African ancestry are more susceptible to CVD, stroke and several autoimmune as well as inflammatory diseases. ${ }^{20} \mathrm{~A}$ better understanding of the role of erythroid ACKR1 in the development of CVDs may help to elucidate pathogenic mechanisms and ultimately to develop therapies specifically tailored to tackle CVD in individuals of African ancestry.

\section{ACKR2}

\section{Receptor Characteristics and Signalling}

ACKR2, also known as D6, was first identified in human lymphatic endothelial cells, ${ }^{21}$ with scavenging properties towards the inflammatory CC chemokines. ${ }^{22}$ Consequently, the role of the atypical receptor in the resolution of inflammation has been extensively studied. ${ }^{23,24}$ ACKR2 has a prominent role in the development of the lymphatic system, ${ }^{25}$ regulating the local microenvironment in lymphatic vessels and cell positioning via one of its ligands CCL2. ${ }^{26}$ Moreover, ACKR2 deficiency in non-haematopoietic cells causes an increase of CCL2 levels in the serum of these mice and a subsequent increase of circulating myeloid cells. ${ }^{27}$ More recently, ACKR2 was also shown to regulate myeloid cell differentiation. ${ }^{28}$

\section{Clinical Significance of ACKR2}

Inflammatory CC chemokines and more specifically CCL2 are key mediators of monocyte infiltration after MI. ${ }^{29,30}$ Cochain et al evaluated the effect of ACKR2 in a mouse model of MI. ${ }^{31}$ In accordance with previous findings, ACKR2 deficiency resulted in elevated levels of CCL2 and CCL3 in the heart of infarcted mice. In addition, deletion of ACKR2 in non-haematopoietic cells enhanced the infiltration of inflammatory monocytes and neutrophils into the heart 5 days after the operation, a process crucial for the healing of the infarcted tissue. ${ }^{32}$ Finally, a fourfold increase of cardiac rupture events was noted in Ackr2 ${ }^{-1-}$ mice. ${ }^{31}$ These data underline the importance of the ACKR2 in inflammation, although further research is needed to elucidate the role of this atypical receptor in CVDs.

\section{ACKR3}

\section{Receptor Characteristics and Signalling}

ACKR3 is highly expressed in the heart, brain, spleen, kidney, lungs and thyroid, ${ }^{8}$ especially by endothelial cells, neurons and marginal B cells. It has an indispensable importance in the development of cardiovascular system, as its absence was shown to result in cardiac phenotype defects, such as heart valve malformation leading to perinatal death in mice. ${ }^{33,34}$ The receptor was first identified from a dog thyroid and formerly known as RDC $1 .{ }^{35}$ Later, it was renamed CXCR7 as a member of the CXC chemokine receptor family. Currently, this receptor is classified as ACKR $3,{ }^{36}$ which is activated by two chemokine ligands, CXCL12 and CXCL11, which furthermore bind CXCR4 and CXCR3, respectively. ${ }^{37}$ However, both ACKR3 and CXCL12 bind each other with greater affinities in comparison to their alternative ligand and receptor, respectively. ${ }^{38-40}$ ACKR3 was described to be internalized upon CXCL12 binding, which ultimately leads to the lysosomal degradation of its bound ligand, ${ }^{39,41-43}$ indicating a scavenging feature. As such, ACKR3 regulates extracellular CXCL12 concentrations ${ }^{44,45}$ and can thereby control CXCR4 activity. ${ }^{46}$ ACKR3, like the other ACKRs, is not able to directly mediate G-protein signalling by itself, nevertheless it may indirectly modulate the G-protein signalling by forming heterodimers with CXCR4. ${ }^{47}$ Moreover, ACKR3 can also recruit and signal through $\beta$-arrestin 2 , an adapter protein involved in receptor signalling and desensitization. ${ }^{48-52}$ This type of signalling was shown to support cell survival and proliferation via Akt activation. ${ }^{53}$ Heinrich et al ${ }^{54}$ have moreover confirmed extracellular signal-regulated kinase $1 / 2$ (ERK1/2) phosphorylation via CXCL12/ACKR3 mediated $\beta$ arrestin 2 signalling. In addition to its well-recognized chemokine ligands CXCL11 and CXCL12, macrophage inhibitory factor (MIF) was recently acknowledged to bind to ACKR $3^{55}$ and mediate phosphoinositide-3-kinase-Akt signalling. ${ }^{56}$ In addition, MIF/ACKR3 interaction has been shown to result in the activation of the ERK1/2 pathway and receptor internalization. ${ }^{57}$ Moreover, a vasodilator hormone named adrenomedullin delivering essential support in cardiac development was also proposed as a ligand for ACKR $3,{ }^{10,58}$ although functional consequences remain to be further elucidated.

\section{Clinical Significance of ACKR3}

Expression of the main ACKR3 ligands, CXCL11 and CXCL12, is reported to be up-regulated under a wide range of pathological conditions; CXCL12 protein expression rises with inflammation, hypoxia as well as ischaemia, ${ }^{59,60}$ whereas CXCL11 expression increases during infection. ${ }^{55}$ Genome-wide association studies marked a strong association between CXCL12 gene locus and CVDs, giving ACKR3 a prominent spot in CVD research. ${ }^{61}$ Several studies already revealed noteworthy effects of ACKR3 in CVDs. Li et al ${ }^{44}$ developed a wire-induced carotid artery injury model in mice with ubiquitous genetic ablation of ACKR3 to study its role in atherosclerosis. Their results showed significant increases in blood cholesterol due to reduced cholesterol uptake by adipose tissue along with advanced atherosclerotic lesions in mice. These findings clearly indicate an atheroprotective role of ACKR3 through lowering blood cholesterol levels and preventing hyperlipidaemia.

Recently, a study by Hao et al ${ }^{62}$ demonstrated a protective role of endothelial ACKR3 in vascular and cardiac remodelling after MI on account of its role in endothelial cell proliferation and angiogenesis. The latter complements previously established roles of ACKR3 in the vascular endothelium presented by Dai et al. ${ }^{63}$ According to their results, the interaction of both ACKR3 and CXCR4 with CXCL12 similarly accounts for proliferation and transmigration of endothelial progenitor cells (EPCs), although ACKR3 is especially responsible for the survival of EPCs as well as the adhesion of these cells onto the 
endothelium. These findings were later confirmed by Yan et $\mathrm{al}^{64}$ (except for the role of ACKR3 in EPC proliferation). EPCs are fundamental regulators of neovascularization in response to tissue ischaemia. ${ }^{65}$ While these and other studies underline a crucial role of ACKR3 in endothelial cells and highlight the therapeutic potential of ACKR3 in angiogenesis, ${ }^{66,67}$ other findings demonstrated a function of ACKR3 on vascular smooth muscle cells (VSMCs).

VSMC migration is an important process during atherosclerotic plaque development necessary to initiate fibrous cap formation, which stabilizes plaques and decreases the risk of plaque rupture and subsequent thrombosis. Interestingly, ACKR3 was described to induce VSMC migration in response to CXCL11 as a result of ACKR3 and $\beta$-arrestin 2 interaction. ${ }^{49}$ This discovery is in sharp contrast to the notion that ACKR3 is not involved in chemotaxis due to its incapacity of G-protein signalling. ${ }^{49}$ Hence, a detailed study investigating the role of VSMC-specific ACKR3 in the onset of atherosclerosis would be of great benefit to CVD research. This would also be a significant addition to the novel role of ACKR3 in the management of thrombosis: MIF-mediated Akt signalling through ACKR3 was shown to decrease apoptosis and promote survival in platelets, ${ }^{56}$ which could have a crucial impact on CVDs offering an outstanding therapeutic potential.

Although these studies clearly demonstrated a protective role of ACKR3 in CVDs, there are also indications of its potential detrimental effects. A key event driving foam cell formation in atherosclerotic lesions is monocyte to macrophage differentiation. Ma et al detected ACKR3 in the macrophage-positive area of aortic atheroma of $A p o e^{-l-}$ mice, but not in healthy aorta. ${ }^{68}$ Further research demonstrated influence of ACKR3 on inflammation, a fundamental process aggravating atherosclerosis. ${ }^{69-71}$ Finally, ACKR3-driven cell adhesion $^{38,63,64,72}$ may be another pro-atherosclerotic event as it might enhance leukocyte adhesion onto the endothelium and thus transmigration and influx into atherosclerotic lesions. These theories yet remain to be elucidated for a better understanding of the specific roles of ACKR3 in CVDs.

The precise impact of ACKR3 in health and disease is a topic under debate due to its wide array of functions. Nevertheless, it is evident that ACKR3 is a significant modulator in CVDs, although a unique function to ACKR3 cannot be assigned, as it has numerous functions in different cell types which reflect great discrepancies depending on the disease model.

\section{ACKR4}

\section{Receptor Characteristics and Signalling}

ACKR4 binds the homeostatic chemokines CCL19, CCL21, CCL25 and CXCL13 and has been attributed scavenging properties. ${ }^{73}$ Few studies investigated the functions of ACKR4 in vivo and it has been proposed that ACKR4 plays a crucial role for the compartmentalization of CCL21 chemokines in lymph nodes. Ulvmar et al reported a selective expression of ACKR4 in lymphatic endothelial cells of the outer wall of lymph node cortex. Such expression establishes a gradient of the chemokine CCL21 between the sub-capsular sinus and the paracortex area. ${ }^{74}$ Consequently, CCR7 positive dendritic cells following this CCL21 gradient entered the lymph node parenchyma. These findings suggest a critical role of ACKR4 in cell chemotaxis into lymph nodes.

\section{Clinical Significance of ACKR4}

The significance of ACKR4 in CVDs has so far not been investigated. However, given that CCR7 positive cells play an important role in atherosclerosis ${ }^{6}$ and that $\mathrm{CXCL} 13$ and CCL21 were shown to be highly expressed and important chemokines for lymphocytes clustering in TLOs, ${ }^{18,75,76}$ it would be interesting to determine the role of ACKR4 in the formation of ATLOs.

\section{Conclusion}

Research focusing on the prevention/treatment of CVDs aims to identify molecular players in this complex and multifaceted disease with the ultimate goal to develop suitable drugs to prevent or treat cardiac diseases. While 'classical' chemokine receptors have been extensively studied in CVDs during the last two decades, the roles of ACKRs in CVDs are still only poorly understood. Therefore, more efforts are needed to better understand ACKRs functions in CVDs, thereby paving the way towards new therapeutic targets.

\section{Note}

After the acceptance of the current manuscript, a study by Girbl et al demonstrating a new function for ACKR1 in leukocyte trafficking was published. This study showed that ACKR1, not only is involved in leukocyte adhesion ${ }^{11}$, but can also regulate leukocyte transendothelial migration. Indeed, CXCL2 produced by neutrophils is deposited on ACKR1 at endothelial junctions, and ACKR1-presented CXCL2 facilitates unidirectional luminal-to-abluminal migration of neutrophils. ${ }^{95}$

\section{Funding}

The authors' research is supported by the DFG (SFB1123 TPA1) to Y.D. and C.W., by the DFG (SFB1123 TPA10) to J.D. and C.W. and by the Alexander von Humboldt Foundation to E.P.C.v.d.V.

Conflict of Interest

None declared.

\section{References}

1 Bäck M, Weber C, Lutgens E. Regulation of atherosclerotic plaque inflammation. J Intern Med 2015;278(05):462-482

2 Hansson GK, Libby P, Tabas I. Inflammation and plaque vulnerability. J Intern Med 2015;278(05):483-493

3 Koenen RR, Weber C. Therapeutic targeting of chemokine interactions in atherosclerosis. Nat Rev Drug Discov 2010;9(02):141-153

4 Dahlöf B. Cardiovascular disease risk factors: epidemiology and risk assessment. Am J Cardiol 2010;105(1, Suppl):3A-9A

5 López-Cotarelo P, Gómez-Moreira C, Criado-García O, Sánchez L, Rodríguez-Fernández JL. Beyond chemoattraction: multifunctionality of chemokine receptors in leukocytes. Trends Immunol 2017;38(12):927-941 
6 van der Vorst EPC, Döring Y, Weber C. Chemokines and their receptors in atherosclerosis. J Mol Med (Berl) 2015;93(09):963-971

7 Ulvmar MH, Hub E, Rot A. Atypical chemokine receptors. Exp Cell Res 2011;317(05):556-568

8 Sánchez-Martín L, Sánchez-Mateos P, Cabañas C. CXCR7 impact on CXCL12 biology and disease. Trends Mol Med 2013;19(01):12-22

9 Benredjem B, Girard M, Rhainds D, St-Onge G, Heveker N. Mutational analysis of atypical chemokine receptor 3 (ACKR3/CXCR7) interaction with its chemokine ligands CXCL11 and CXCL12.J Biol Chem 2017;292(01):31-42

10 Betterman KL, Harvey NL. Decoys and cardiovascular development: CXCR7 and regulation of adrenomedullin signaling. Dev Cell 2014;30(05):490-491

11 Pruenster M, Mudde L, Bombosi P, et al. The Duffy antigen receptor for chemokines transports chemokines and supports their promigratory activity. Nat Immunol 2009;10(01):101-108

12 Novitzky-Basso I, Rot A. Duffy antigen receptor for chemokines and its involvement in patterning and control of inflammatory chemokines. Front Immunol 2012;3:266

13 Howes RE, Patil AP, Piel FB, et al. The global distribution of the Duffy blood group. Nat Commun 2011;2:266

14 Tournamille C, Colin Y, Cartron JP, Le Van Kim C. Disruption of a GATA motif in the Duffy gene promoter abolishes erythroid gene expression in Duffy-negative individuals. Nat Genet 1995;10(02):224-228

15 Duchene J, Novitzky-Basso I, Thiriot A, et al. Atypical chemokine receptor 1 on nucleated erythroid cells regulates hematopoiesis. Nat Immunol 2017;18(07):753-761

16 Wan W, Liu Q, Lionakis MS, et al. Atypical chemokine receptor 1 deficiency reduces atherogenesis in ApoE-knockout mice. Cardiovasc Res 2015;106(03):478-487

17 Thiriot A, Perdomo C, Cheng G, et al. Differential DARC/ACKR1 expression distinguishes venular from non-venular endothelial cells in murine tissues. BMC Biol 2017;15(01):45

18 Hu D, Mohanta SK, Yin C, et al. Artery tertiary lymphoid organs control aorta immunity and protect against atherosclerosis via vascular smooth muscle cell lymphotoxin $\beta$ receptors. Immunity 2015;42(06):1100-1115

19 Unruh D, Srinivasan R, Benson T, et al. Red blood cell dysfunction induced by high-fat diet: potential implications for obesityrelated atherosclerosis. Circulation 2015;132(20):1898-1908

20 Carnethon MR, Pu J, Howard G, et al; American Heart Association Council on Epidemiology and Prevention; Council on Cardiovascular Disease in the Young; Council on Cardiovascular and Stroke Nursing; Council on Clinical Cardiology; Council on Functional Genomics and Translational Biology; and Stroke Council. Cardiovascular health in African Americans: a scientific statement from the American Heart Association. Circulation 2017;136(21):e393-e423

21 Nibbs RJ, Wylie SM, Pragnell IB, Graham GJ. Cloning and characterization of a novel murine beta chemokine receptor, D6. Comparison to three other related macrophage inflammatory protein-1alpha receptors, CCR-1, CCR-3, and CCR-5. J Biol Chem 1997;272(19):12495-12504

22 Fra AM, Locati M, Otero K, et al. Cutting edge: scavenging of inflammatory CC chemokines by the promiscuous putatively silent chemokine receptor D6. J Immunol 2003;170(05):2279-2282

23 Graham GJ. D6/ACKR2. Front Immunol 2015;6:280

24 Bonecchi R, Garlanda C, Mantovani A, Riva F. Cytokine decoy and scavenger receptors as key regulators of immunity and inflammation. Cytokine 2016;87:37-45

25 Bonavita O, Mollica Poeta V, Setten E, Massara M, Bonecchi R. ACKR2: an atypical chemokine receptor regulating lymphatic biology. Front Immunol 2017;7:691

26 Lee KM, Danuser R, Stein JV, Graham D, Nibbs RJ, Graham GJ. The chemokine receptors ACKR2 and CCR2 reciprocally regulate lymphatic vessel density. EMBO J 2014;33(21):2564-2580

27 Savino B, Castor MG, Caronni N, et al. Control of murine Ly6C (high) monocyte traffic and immunosuppressive activities by atypical chemokine receptor D6. Blood 2012;119(22):5250-5260
28 Massara M, Bonavita O, Savino B, et al. ACKR2 in hematopoietic precursors as a checkpoint of neutrophil release and anti-metastatic activity. Nat Commun 2018;9(01):676

29 Dutta P, Nahrendorf M. Monocytes in myocardial infarction. Arterioscler Thromb Vasc Biol 2015;35(05):1066-1070

30 Schloss MJ, Hilby M, Nitz K, et al. Ly6C ${ }^{\text {high }}$ monocytes oscillate in the heart during homeostasis and after myocardial infarctionbrief report. Arterioscler Thromb Vasc Biol 2017;37(09): 1640-1645

31 Cochain C, Auvynet C, Poupel L, et al. The chemokine decoy receptor D6 prevents excessive inflammation and adverse ventricular remodeling after myocardial infarction. Arterioscler Thromb Vasc Biol 2012;32(09):2206-2213

32 Horckmans M, Ring L, Duchene J, et al. Neutrophils orchestrate postmyocardial infarction healing by polarizing macrophages towards a reparative phenotype. Eur Heart J 2017;38(03):187-197

33 Sierro F, Biben C, Martínez-Muñoz L, et al. Disrupted cardiac development but normal hematopoiesis in mice deficient in the second CXCL12/SDF-1 receptor, CXCR7. Proc Natl Acad Sci U S A 2007;104 (37):14759-14764

34 Gerrits H, van Ingen Schenau DS, Bakker NE, et al. Early postnatal lethality and cardiovascular defects in CXCR7-deficient mice. Genesis 2008;46(05):235-245

35 Libert F, Parmentier M, Lefort A, et al. Selective amplification and cloning of four new members of the $G$ protein-coupled receptor family. Science 1989;244(4904):569-572

36 Graham GJ, Locati M, Mantovani A, Rot A, Thelen M. The biochemistry and biology of the atypical chemokine receptors. Immunol Lett 2012;145(1-2):30-38

37 Gustavsson M, Wang L, van Gils N, et al. Structural basis of ligand interaction with atypical chemokine receptor 3. Nat Commun 2017;8:14135

38 Burns JM, Summers BC, Wang Y, et al. A novel chemokine receptor for SDF-1 and I-TAC involved in cell survival, cell adhesion, and tumor development. J Exp Med 2006;203(09):2201-2213

39 Balabanian K, Lagane B, Infantino S, et al. The chemokine SDF-1/ CXCL12 binds to and signals through the orphan receptor RDC1 in T lymphocytes. J Biol Chem 2005;280(42):35760-35766

40 Crump MP, Gong JH, Loetscher P, et al. Solution structure and basis for functional activity of stromal cell-derived factor-1; dissociation of CXCR4 activation from binding and inhibition of HIV-1. EMBO J 1997;16(23):6996-7007

41 Luker KE, Steele JM, Mihalko LA, Ray P, Luker GD. Constitutive and chemokine-dependent internalization and recycling of CXCR7 in breast cancer cells to degrade chemokine ligands. Oncogene 2010;29(32):4599-4610

42 Hoffmann F, Müller W, Schütz D, et al. Rapid uptake and degradation of CXCL12 depend on CXCR7 carboxyl-terminal serine/ threonine residues. J Biol Chem 2012;287(34):28362-28377

43 Boldajipour B, Mahabaleshwar H, Kardash E, et al. Control of chemokine-guided cell migration by ligand sequestration. Cell 2008;132(03):463-473

44 Li X, Zhu M, Penfold ME, et al. Activation of CXCR7 limits atherosclerosis and improves hyperlipidemia by increasing cholesterol uptake in adipose tissue. Circulation 2014;129(11):1244-1253

45 Naumann U, Cameroni E, Pruenster M, et al. CXCR7 functions as a scavenger for CXCL12 and CXCL11. PLoS One 2010;5(02):e9175

46 Thelen M, Thelen S. CXCR7, CXCR4 and CXCL12: an eccentric trio? J Neuroimmunol 2008;198(1-2):9-13

47 Uto-Konomi A, McKibben B, Wirtz J, et al. CXCR7 agonists inhibit the function of CXCL12 by down-regulation of CXCR4. Biochem Biophys Res Commun 2013;431(04):772-776

48 Coggins NL, Trakimas D, Chang SL, et al. CXCR7 controls competition for recruitment of $\beta$-arrestin 2 in cells expressing both CXCR4 and CXCR7. PLoS One 2014;9(06):e98328

49 Rajagopal S, Kim J, Ahn S, et al. Beta-arrestin- but not G proteinmediated signaling by the "decoy" receptor CXCR7. Proc Natl Acad Sci U S A 2010;107(02):628-632 
50 Kalatskaya I, Berchiche YA, Gravel S, Limberg BJ, Rosenbaum JS, Heveker N. AMD3100 is a CXCR7 ligand with allosteric agonist properties. Mol Pharmacol 2009;75(05):1240-1247

51 Chen D, Xia Y, Zuo K, et al. Crosstalk between SDF-1/CXCR4 and SDF-1/CXCR7 in cardiac stem cell migration. Sci Rep 2015; $5: 16813$

52 Chen Q Zhang M, Li Y, et al. CXCR7 mediates neural progenitor cells migration to CXCL12 independent of CXCR4. Stem Cells 2015;33(08):2574-2585

53 Torossian F, Anginot A, Chabanon A, et al. CXCR7 participates in CXCL12-induced CD34+ cell cycling through $\beta$-arrestin-dependent Akt activation. Blood 2014;123(02):191-202

54 Heinrich EL, Lee W, Lu J, Lowy AM, Kim J. Chemokine CXCL12 activates dual CXCR4 and CXCR7-mediated signaling pathways in pancreatic cancer cells. J Transl Med 2012;10:68

55 Wang C, Chen W, Shen J. CXCR7 targeting and its major disease relevance. Front Pharmacol 2018;9:641

56 Chatterjee M, von Ungern-Sternberg SN, Seizer P, et al. Plateletderived CXCL12 regulates monocyte function, survival, differentiation into macrophages and foam cells through differential involvement of CXCR4-CXCR7. Cell Death Dis 2015;6:e1989

57 Alampour-Rajabi S, El Bounkari O, Rot A, et al. MIF interacts with CXCR7 to promote receptor internalization, ERK1/2 and ZAP-70 signaling, and lymphocyte chemotaxis. FASEB J 2015;29(11):4497-4511

58 Kapas S, Clark AJ. Identification of an orphan receptor gene as a type 1 calcitonin gene-related peptide receptor. Biochem Biophys Res Commun 1995;217(03):832-838

59 Karin N. The multiple faces of CXCL12 (SDF-1alpha) in the regulation of immunity during health and disease. J Leukoc Biol 2010;88(03):463-473

$60 \mathrm{Li}$ M, Ransohoff RM. The roles of chemokine CXCL12 in embryonic and brain tumor angiogenesis. Semin Cancer Biol 2009;19(02):111-115

61 Samani NJ, Erdmann J, Hall AS, et al; WTCCC and the Cardiogenics Consortium. Genome-wide association analysis of coronary artery disease. N Engl J Med 2007;357(05):443-453

62 Hao H, Hu S, Chen H, et al. Loss of endothelial CXCR7 impairs vascular homeostasis and cardiac remodeling after myocardial infarction: implications for cardiovascular drug discovery. Circulation 2017;135(13):1253-1264

63 Dai X, Tan Y, Cai S, et al. The role of CXCR7 on the adhesion, proliferation and angiogenesis of endothelial progenitor cells. J Cell Mol Med 2011;15(06):1299-1309

64 Yan X, Cai S, Xiong X, et al. Chemokine receptor CXCR7 mediates human endothelial progenitor cells survival, angiogenesis, but not proliferation. J Cell Biochem 2012;113(04):1437-1446

65 Urbich C, Dimmeler S. Endothelial progenitor cells: characterization and role in vascular biology. Circ Res 2004;95(04):343-353

66 Dai X, Yan X, Zeng J, et al. Elevating CXCR7 improves angiogenic function of EPCs via Akt/GSK-3ß/Fyn-mediated Nrf2 activation in diabetic limb ischemia. Circ Res 2017;120(05):e7-e23

67 Cao Z, Tong X, Xia W, et al. CXCR7/p-ERK-signaling is a novel target for therapeutic vasculogenesis in patients with coronary artery disease. PLoS One 2016;11(09):e0161255

68 Ma W, Liu Y, Ellison N, Shen J. Induction of C-X-C chemokine receptor type 7 (CXCR7) switches stromal cell-derived factor-1 (SDF-1) signaling and phagocytic activity in macrophages linked to atherosclerosis. J Biol Chem 2013;288(22):15481-15494

69 Ngamsri K-C, Müller A, Bösmüller H, Gamper-Tsigaras J, Reutershan J, Konrad FM. The pivotal role of CXCR7 in stabilization of the pulmonary epithelial barrier in acute pulmonary inflammation. J Immunol 2017;198(06):2403-2413

70 Peng H, Zhang H, Zhu H. Blocking CXCR7-mediated adipose tissue macrophages chemotaxis attenuates insulin resistance and inflammation in obesity. Biochem Biophys Res Commun 2016; 479(04):649-655

71 Chang H-C, Huang PH, Syu FS, et al. Critical involvement of atypical chemokine receptor CXCR7 in allergic airway inflammation. Immunology 2018;154(02):274-284
72 Mazzinghi B, Ronconi E, Lazzeri E, et al. Essential but differential role for CXCR4 and CXCR7 in the therapeutic homing of human renal progenitor cells. J Exp Med 2008;205(02):479-490

73 Comerford I, Milasta S, Morrow V, Milligan G, Nibbs R. The chemokine receptor CCX-CKR mediates effective scavenging of CCL19 in vitro. Eur J Immunol 2006;36(07):1904-1916

74 Ulvmar MH, Werth K, Braun A, et al. The atypical chemokine receptor CCRL1 shapes functional CCL21 gradients in lymph nodes. Nat Immunol 2014;15(07):623-630

75 Horckmans M, Bianchini M, Santovito D, et al. Pericardial adipose tissue regulates granulopoiesis, fibrosis, and cardiac function after myocardial infarction. Circulation 2018;137(09):948-960

76 Bénézech C, Luu NT, Walker JA, et al. Inflammation-induced formation of fat-associated lymphoid clusters. Nat Immunol 2015;16(08):819-828

77 Zlotnik A, Yoshie O. The chemokine superfamily revisited. Immunity 2012;36(05):705-716

78 Bachelerie F, Ben-Baruch A, Burkhardt AM, et al. International Union of Basic and Clinical Pharmacology. [corrected]. LXXXIX. Update on the extended family of chemokine receptors and introducing a new nomenclature for atypical chemokine receptors. Pharmacol Rev 2013;66(01):1-79

79 Schneider EH, Fowler SC, Lionakis MS, et al. Regulation of motor function and behavior by atypical chemokine receptor 1 . Behav Genet 2014;44(05):498-515

80 Neote K, Mak JY, Kolakowski LF Jr, Schall TJ. Functional and biochemical analysis of the cloned Duffy antigen: identity with the red blood cell chemokine receptor. Blood 1994;84(01): 44-52

81 Chakera A, Seeber RM, John AE, Eidne KA, Greaves DR. The Duffy antigen/receptor for chemokines exists in an oligomeric form in living cells and functionally antagonizes CCR5 signaling through hetero-oligomerization. Mol Pharmacol 2008;73(05): 1362-1370

82 Vinet J, van Zwam M, Dijkstra IM, et al. Inhibition of CXCR3mediated chemotaxis by the human chemokine receptor-like protein CCX-CKR. Br J Pharmacol 2013;168(06):1375-1387

83 Nibbs RJ, Kriehuber E, Ponath PD, et al. The beta-chemokine receptor D6 is expressed by lymphatic endothelium and a subset of vascular tumors. Am J Pathol 2001;158(03):867-877

84 Hansell CAH, Hurson CE, Nibbs RJB. DARC and D6: silent partners in chemokine regulation? Immunol Cell Biol 2011;89(02): 197-206

85 Gardner L, Patterson AM, Ashton BA, Stone MA, Middleton J. The human Duffy antigen binds selected inflammatory but not homeostatic chemokines. Biochem Biophys Res Commun 2004; 321(02):306-312

86 Bachelerie F, Graham GJ, Locati M, et al. New nomenclature for atypical chemokine receptors. Nat Immunol 2014;15(03): 207-208

87 Bryce SA, Wilson RA, Tiplady EM, et al. ACKR4 on stromal cells scavenges CCL19 to enable CCR7-dependent trafficking of APCs from inflamed skin to lymph nodes. J Immunol 2016;196(08): 3341-3353

88 Thomson CA, van de Pavert SA, Stakenborg M, et al. Expression of the atypical chemokine receptor ACKR4 identifies a novel population of intestinal submucosal fibroblasts that preferentially expresses endothelial cell regulators. J Immunol 2018;201(01): 215-229

89 Puddinu V, Casella S, Radice E, et al. ACKR3 expression on diffuse large B cell lymphoma is required for tumor spreading and tissue infiltration. Oncotarget 2017;8(49):85068-85084

90 Salvi V, Sozio F, Sozzani S, Del Prete A. Role of atypical chemokine receptors in microglial activation and polarization. Front Aging Neurosci 2017;9:148

91 Berahovich RD, Zabel BA, Lewén S, et al. Endothelial expression of CXCR7 and the regulation of systemic CXCL12 levels. Immunology 2014;141(01):111-122 
92 Borroni EM, Cancellieri C, Vacchini A, et al. $\beta$-arrestin-dependent activation of the cofilin pathway is required for the scavenging activity of the atypical chemokine receptor D6. Sci Signal 2013;6 (273, ra30)1-11, S1-S3

93 Bonecchi R, Borroni EM, Anselmo A, et al. Regulation of D6 chemokine scavenging activity by ligand- and Rab11-dependent surface up-regulation. Blood 2008;112(03):493-503
94 Watts AO, Verkaar F, van der Lee MM, et al. $\beta$-Arrestin recruitment and $G$ protein signaling by the atypical human chemokine decoy receptor CCX-CKR. J Biol Chem 2013;288(10):7169-7181

95 Girbl T, Lenn T, Perez L, et al. Distinct compartmentalization of the chemokines CXCL1 and CXCL2 and the atypical receptor ACKR1 determine discrete stages of neutrophil diapedesis. Immunity 2018;49(06):1062-1076 